\section{Switchable Supramolecular Organization of Structurally Defined Micelles Based on an Amphiphilic Fullerene**}

\section{Stephan Burghardt, Andreas Hirsch,* Boris Schade, Kai Ludwig, and Christoph Böttcher*}

Some time ago we started to investigate the synthesis and supramolecular organization of dendritic amphiphiles that contain fullerenes or calixarenes as core units..$^{[1-3]}$ Apart from the synthesis of structurally persistent micelles, we were particularly interested in the assembly of functionalized micelles and liposomes in order to direct the aggregation behavior of these species with an appropriate external stimulus. Results from these studies could be useful for the development of drug-delivery systems. Examples of such dendritic amphiphiles include the globular amphifullerene 1, ${ }^{[1]}$ which contains a pair of carboxy-terminated amide dendrons and five dodecyl malonate addends attached to the fullerene core in an octahedral [1:5] addition pattern, and the calixarene derivative $\mathbf{2}^{[3]}$ which contains analogous hydrophilic and lipophilic units. In amphiphile $\mathbf{1}$ the space required for the hydrophilic and the hydrophobic areas is balanced whereas the extended chains have free conformational mobility. This ensures a wide spectrum of energetically favorable packing motifs both for the formation of micellar structures and for extended liposomal double layers. The existence of different aggregates was confirmed by electron microscopic investigations. ${ }^{[2 a]}$

By contrast, we designed amphiphile $\mathbf{2}$ as a comparatively rigid T-shaped structure $^{[3]}$ that does not pack to form extended ultrastructures such as liposomes. We demonstrated that the supramolecular organization of $\mathbf{2}$ yields very small and strongly bent aggregates exclusively, which are the first proven examples of structurally persistent micelles. ${ }^{[3]}$ Each of these $C_{2}$-symmetrical micelles consists of exactly seven molecules of amphiphile $\mathbf{2}$. The structure was determined by cryogenic transmission electron microscopy (cryo-TEM) and

[*] S. Burghardt, Prof. Dr. A. Hirsch Institut für Organische Chemie Universität Erlangen-Nürnberg Henkestrasse 42, 91054 Erlangen (Germany) Fax: (+49) 9131-852-6864

E-mail: andreas.hirsch@chemie.uni-erlangen.de

Dr. B. Schade, Dr. K. Ludwig, Dr. C. Böttcher

Forschungszentrum für Elektronenmikroskopie Institut für Chemie

Freie Universität Berlin

Fabeckstrasse 36a, 14195 Berlin (Germany)

Fax: $(+49)$ 30-838-56589

E-mail: bottcher@chemie.fu-berlin.de

[**] The authors are grateful to the Deutsche Forschungsgemeinschaft for financial support (DFG AH468/13-1 and Großgeräteinitiative to FU Berlin).

(9) upporting information for this article is available on the WWW under http://www.angewandte.org or from the author.
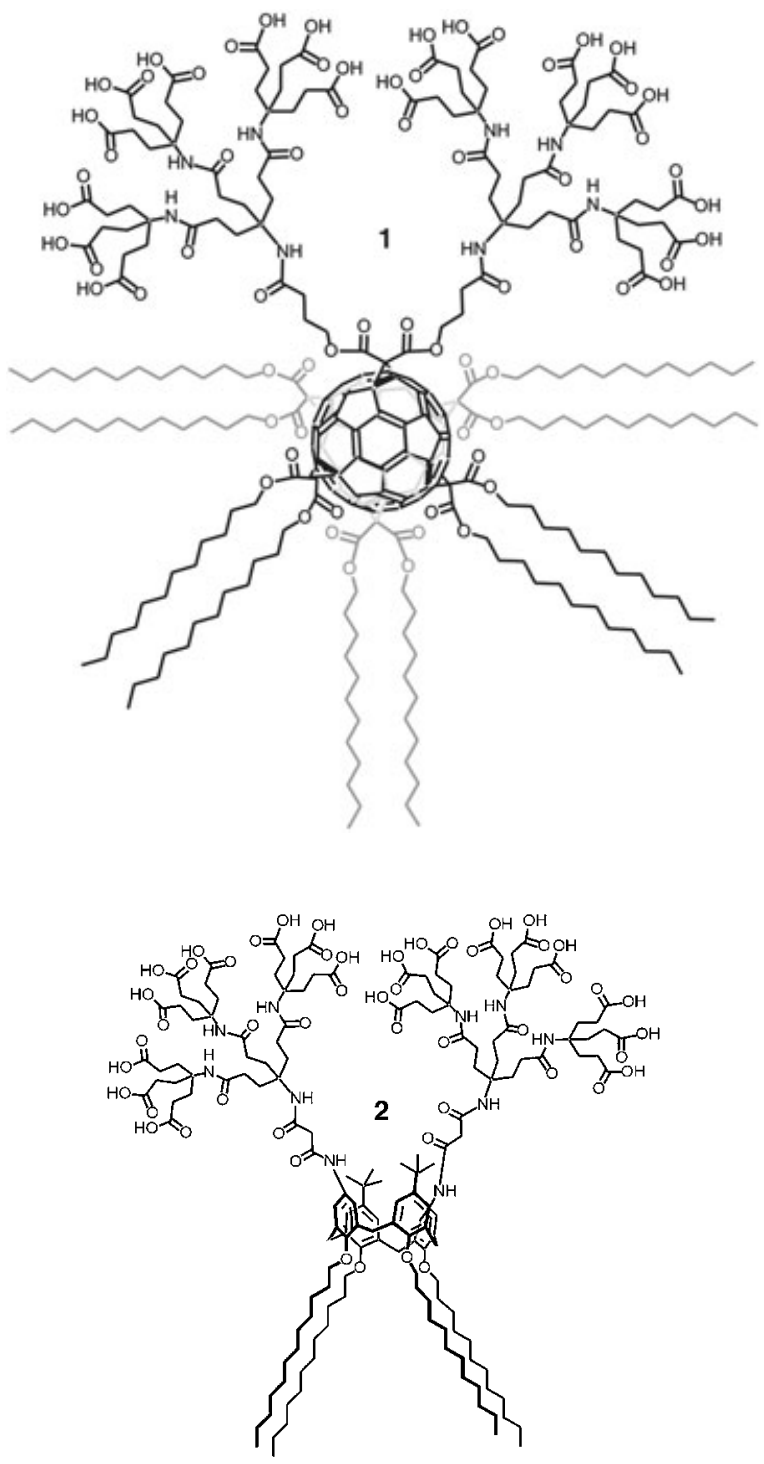

techniques for the three-dimensional reconstruction of single particles at a resolution of $12 \AA^{[3]}$

We report here for the first time on amphiphile $\mathbf{3}$ (see the Supporting Information), whose aggregation in different supramolecular organizational forms may be switched by changing the $\mathrm{pH}$ (Figure 1). In principle, the aggregation behavior of amphiphilic systems may be studied under quasinative conditions by using combined methods: ultrafast cryogenic fixation of the samples ("vitrification") and direct observation by transmission electron microscopy upon simultaneous sample cooling $(\approx 120 \mathrm{~K})$. The cooling rates $\left(>10^{4} \mathrm{~K} \mathrm{~s}^{-1}\right)$ during sample preparation are so high that molecular reorientation (e.g. formation of ice crystals) is prevented. ${ }^{[5,6]}$ Ultimately, projection images of the aggregates are obtained by microscopy and thus information about their intrinsic structure is provided.

We examined amphiphile 3 at neutral $\mathrm{pH}$ in water (phosphate buffer $\mathrm{pH}$ 7.2) by these methods and found predominantly rod-shaped aggregates with a double-layer ultrastructure (Figure $2 \mathrm{a}$ ). The diameter of these rods is $65 \pm$ 

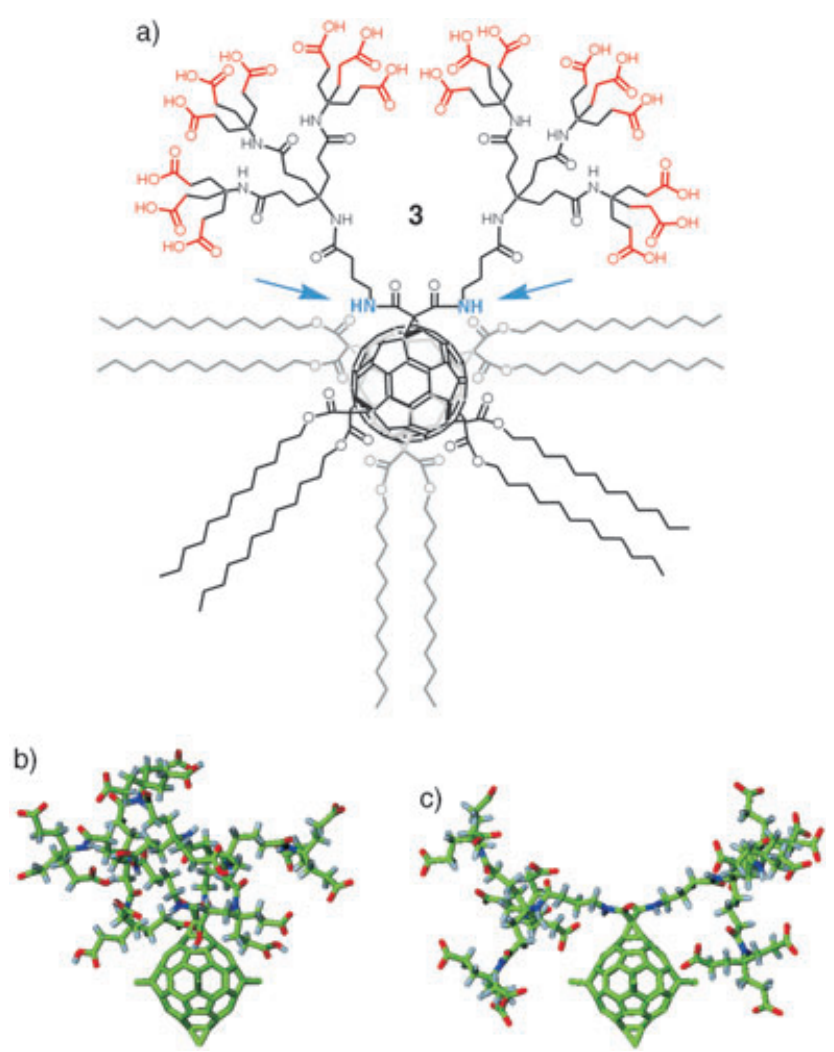

Figure 1. a) Amphiphilic fullerene dendrimer 3 with dendritic branches linked by amide bonds (blue arrow). b, c) Molecular models of differently protonated conformers of $\mathbf{3}$, modeled with the program Tinker. ${ }^{[7]}$ For clarity, the alkyl chains are not depicted. b) Dendrons partially protonated. According to earlier titration measurements on LB films, a $\mathrm{p} K_{\mathrm{a}}$ of 7.5 is found for the chemically related dendrimer $1{ }^{[2 \mathrm{~b}]}$ Accordingly, the simulation of 3 at $\mathrm{pH} 7.2$ has been carried out by using eight negative charges (of a total of possible 18). c) Dendrons completely deprotonated (18 negative charges).
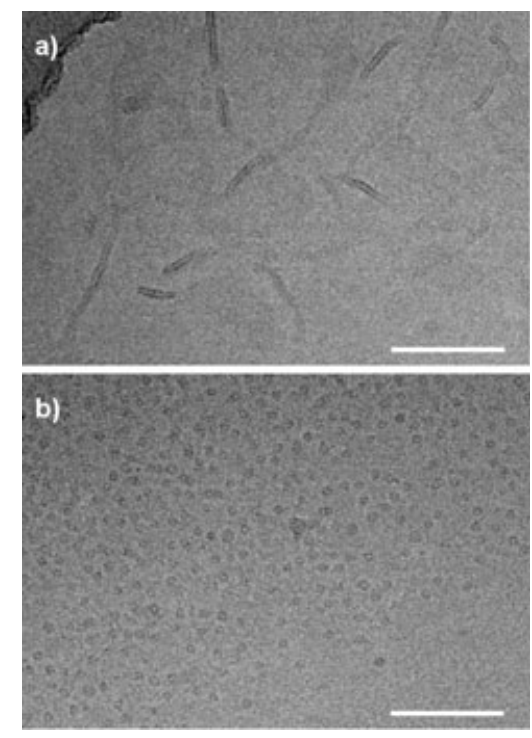

Figure 2. Representative electron micrograph showing aggregates of 3 embedded in vitrified water (scale bar: $50 \AA$ A). a) At pH 7.2 predominantly rod-shaped double-layered aggregates are found. b) At pH 9.2 exclusively globular micelles are found.
$5 \AA$; the length is strongly variable and exceeds the diameter considerably. In addition, a few globular aggregates with a diameter of $85 \pm 10 \AA$ were found. When the $\mathrm{pH}$ of the neutral solution was increased to roughly 9 (borate buffer $\mathrm{pH}$ 9.2), those globular micelles with a diameter of $85 \pm 10 \AA$ were found exclusively (Figure $2 \mathrm{~b}$ ). Interestingly, apart from variations in diameter these globular micelles show a distinct internal structure. By analogy to our investigations on micellar structures of amphiphile $\mathbf{2}^{[3]}$ we considered the structural motifs of the globular micelles to be an indication of structural persistence. Accordingly, each individual structure should represent a distinct spatial orientation of the aggregate. Thus, it should be possible in principle to determine the three-dimensional organization from the image data; variations in the diameters would arise from the aggregates' different orientations. By using the reconstruction techniques described earlier ${ }^{[3]}$ (alignment, classification, angular reconstitution) (Figure $3 \mathrm{a}$ ), we calculated the three-dimensional

a)

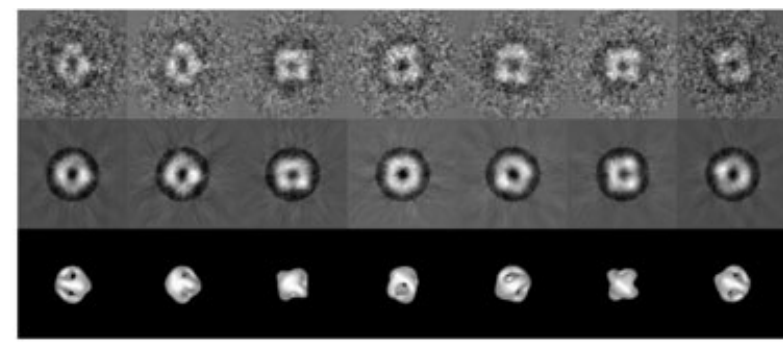

b)
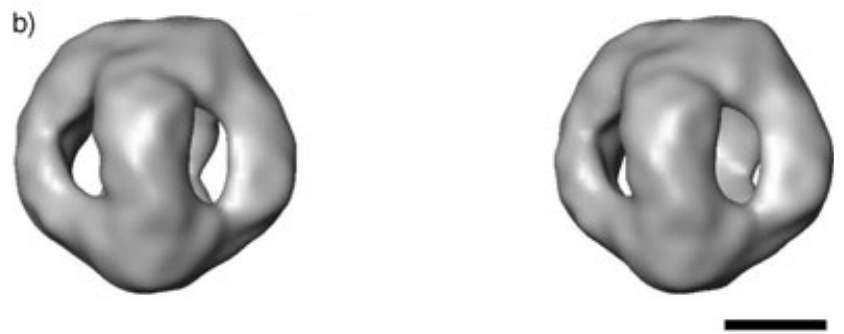

Figure 3. Three-dimensional reconstruction from electron cryogenic microscopic data of globular micelles formed by 3 at $\mathrm{pH}$ 9.2. a) The first row displays class sum images of micelles in various spatial orientations. Three-dimensional structural information was derived from the corresponding Euler angles at a resolution level of $\approx 16 \AA$. Back projections (second row) of the calculated volumes (third row) at identical Euler angles indicate the consistency with the experimental data. b) Stereoscopic view of the final three-dimensional reconstruction (scale bar: $25 \AA$ ).

structure for the globular micelles of $\mathbf{3}$ (Figure $3 \mathrm{~b}$ ). The successful reconstruction is evidence that the spatial molecular organization of the aggregates is identical within the achieved level of resolution ( $\approx 5000$ individual aggregates were randomly extracted from the microscopic data).

To determine the possible molecular organization in the aggregates, we undertook a visual three-dimensional fitting of molecular models of $\mathbf{3}$ into the reconstructed 3D density map (Figure $4 \mathrm{a}$ and $\mathrm{b}$ ). Based on a simulated conformation of $\mathbf{3}$ in its completely deprotonated form (Figure 1c) (generated with the program Tinker ${ }^{[7]}$ ), the globular micellar structure may be 
a)
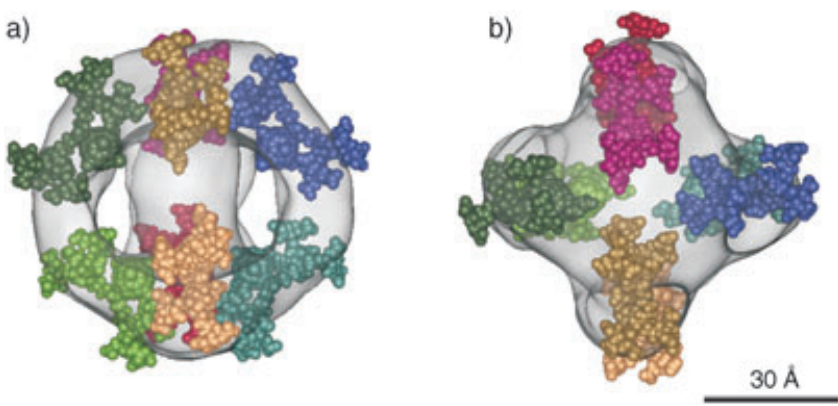

c)

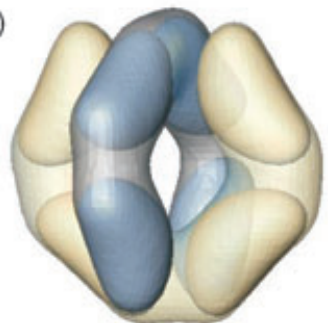

d)

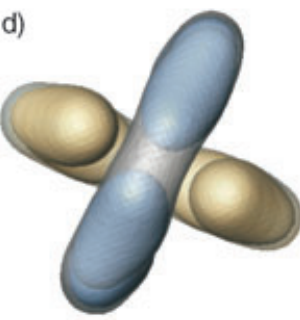

Figure 4. Interpretation of the reconstructed data. a) Eight head groups of the dendritic fullerene molecule may be fitted in a $C_{2}$-symmetrical mode into the reconstructed electron-density map. b) View of (a) tilted by $90^{\circ}$ towards the front side. c) Schematic representation of the head-group assembly: Two planar U-shaped assemblies consisting of four molecules each (yellow and blue) are displaced by $90^{\circ}$ along their longitudinal axes and are inserted — though not completely-into each other at their open ends. d) View of c) tilted by $90^{\circ}$ towards the front side.

constructed from eight molecules in a $C_{2}$-symmetrical arrangement. The molecular organization of this aggregate may be described best by considering two planar U-shaped structures consisting of four molecules each. These are displaced by $90^{\circ}$ along the longitudinal axis with respect to their open ends; however, they are not inserted completely into each other (Figure $4 \mathrm{c}$ and d). This molecular organization ensures a nearly spherical shape overall and thus very efficient shielding of all hydrophobic molecule parts from the aqueous environment. As was the case for the structurally persistent micelles derived from calixarene 2, the innermost core area of the micelle formed by the hydrophobic alkyl chains is not reproduced in the reconstruction. Since individual chains do not assume a spatially defined conformation but are more or less arranged in a statistical way, this part of the aggregate is averaged out as a result of the statistical principles of the applied reconstruction method. Only the head groups, which are located easily due to the high-contrast fullerene entity, prove to be sterically persistent, thus yielding the found structural pattern.

The molecular structure of $\mathbf{3}$ encodes a supramolecular behavior representing a combination of supramolecular properties of $\mathbf{1}$ and $\mathbf{2}$. On the one hand, and similar to $\mathbf{1}$, the spatial demands of the hydrophilic and hydrophobic areas are well-balanced. On the other hand, and similar to 2 , the dendritic branches are linked by amide rather than ester linkages, resulting in enhanced rigidity. The significant structural change when the $\mathrm{pH}$ is lowered may be explained by electrostatic repulsion of the completely deprotonated carboxy groups, and enhanced solvation is a secondary effect.

Accordingly, we may assume that at neutral pH the electrostatic repulsion is reduced as a consequence of partial protonation. This causes reduced solvation, which in turn leads to much closer packing as is evidenced by the smaller diameter of the rods relative to that of the globular micelles. Figure $1 \mathrm{~b}$ shows the corresponding result of a conformational simulation of $\mathbf{3}$ in aqueous environment at neutral $\mathrm{pH}$. The fact that the rod diameter is constant throughout suggests that the three-dimensional organization must be based on a circular-shaped cross-sectional area. Based on the dimensions and density profile, a double-layered molecular arrangement in a micellar cylinder can be visualized. According to this motif an appropriate radius of curvature of ca. $65 \AA$ can be modeled by using approximately eight molecules. The corresponding model is depicted in Figure 5.

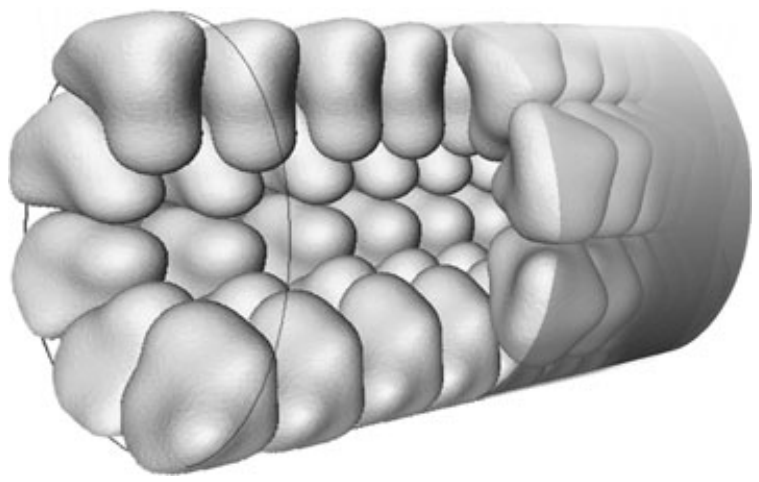

Figure 5. Proposed model of a molecular arrangement of $\mathbf{3}$ in micellar rods at neutral $\mathrm{pH}$. The employed head-group volume is based on the partly protonated form shown in Figure $1 \mathrm{~b}$. Hydrophobic alkyl chains, which should reside at the center of the micelle, are not shown for clarity. They correspond to areas of low density in the electron microscopic images of Figure $2 \mathrm{a}$.

We have demonstrated that by suitable substitution structurally persistent aggregates may be generated in a reproducible way. This opens up perspectives for the systematic investigation of the parameters responsible for aggregate formation. Fundamental investigations on the stability of the aggregates, in particular tolerance of chemical modification and functionalization of the head group, might form the basis for the synthesis of tailor-made transport systems. These may have potential for the selective deposition of drugs in living organisms.

Received: October 29, 2004

Published online: April 13, 2005

Keywords: amphiphiles · dendrimers · electron microscopy · fullerenes $\cdot$ micelles $\cdot$ self-assembly

[1] a) M. Brettreich, A. Hirsch, Tetrahedron Lett. 1998, 39, 2731 2734; b) M. Braun, S. Atalick, D. M. Guldi, H. Lanig, M. Brettreich, S. Burghardt, M. Hatzimarinaki, E. Ravanelli, M. Prato, R. Van Eldik, A. Hirsch, Chem. Eur. J. 2003, 9, 3867-3875.

[2] a) M. Brettreich, S. Burghardt, C. Böttcher, T. Bayerl, S. Bayerl, A. Hirsch, Angew. Chem. 2000, 112, 1915; Angew. Chem. Int. Ed. 
2000, 39, 1845-1848; b) A. P. Maierhofer, M. Brettreich, S. Burghardt, O. Vostrowsky, A. Hirsch, S. Langridge, T. M. Bayerl, Langmuir 2000, 16, 8884-8891.

[3] N. Kellermann, W. Bauer, A. Hirsch, B. Schade, K. Ludwig, C. Böttcher, Angew. Chem. 2004, 116, 3019-3022; Angew. Chem. Int. Ed. 2004, 43, 2959-2962.
[4] A. Hirsch, O. Vostrowsky, Eur. J. Org. Chem. 2001, 829-848.

[5] M. Adrian, J. Dubochet, J. Lepault, A. W. McDowall, Nature 1984, $308,32-36$.

[6] J. Dubochet, M. Adrian, J.-J. Chang, J.-C. Homo, J. Lepault, A. W. McDowall, P. Schultz, Q. Rev. Biophys. 1988, 21, 129-228.

[7] http://dasher.wustl.edu/tinker/.

\section{The definitive work in electrochemistry}

\section{Encyclopedia of Electrochemistry}

Available Volumes:

1: Thermodynamics and Electrified Interfaces

2: Interfacial Kinetics and Mass Transport

3: Instrumentation and Electroanalytical Chemistry

4: Corrosion and Oxide Films

6: Semiconductor Electrodes and Photoelectrochemistry

8: Organic Electrochemistry

9: Bioelectrochemistry

Forthcoming Volumes (completed May 2006):

5: Electrochemical Engineering

7: Inorganic Electrochemistry

10: Modified Electrodes

11: Index

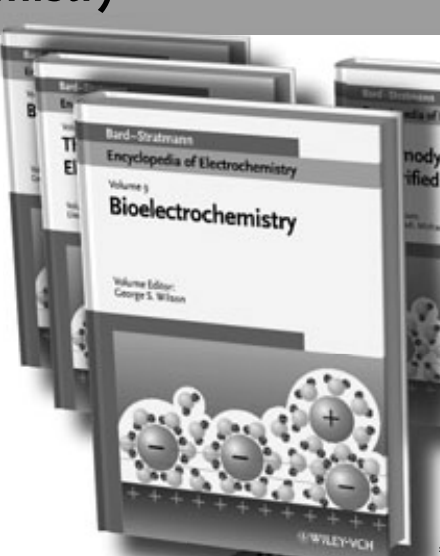

Editors-in-Chief: Allen J. Bard, Department of Chemistry, University of Texas, Austin, USA/ Martin Stratmann, MaxPlanck-Institute for Iron Research, Duesseldorf, Germany

Stay up-to-date in electrochemistry

- a total of 11 volumes makes this the first and only complete reference on electrochemistry

- covering all aspects, from fundamental research to applications in industry

$\vec{b}$ easy access to electrochemical topics

敨

www.wiley-vch.de/bard/eoe 